\title{
MicroRNA-125a-3p downregulation correlates with tumorigenesis and poor prognosis in patients with non-small cell lung cancer
}

\author{
LIKUN HOU $^{1 *}$, PEI LUO ${ }^{2 *}$, YUSHUI MA ${ }^{3 *}$, CHENGYOU JIA $^{3}$, FEI YU ${ }^{3}$, ZHONGWEI LV $^{3}$, \\ CHUNYAN WU ${ }^{1}$ and DA FU ${ }^{3,4}$ \\ ${ }^{1}$ Department of Pathology, Shanghai Pulmonary Hospital, Tongji University School of Medicine, Shanghai 200433; \\ ${ }^{2}$ Veterinary Faculty, College of Veterinary Medicine, Hunan Agricultural University, Changsha, Hunan 410128; \\ ${ }^{3}$ Department of Nuclear Medicine; ${ }^{4}$ Central Laboratory for Medical Research, Shanghai 10th People's Hospital, \\ Tongji University School of Medicine, Shanghai 200072, P.R. China
}

Received April 28, 2016; Accepted December 20, 2016

DOI: $10.3892 / 01.2017 .6809$

\begin{abstract}
MicroRNA (miR)-125a-3p is derived from the 3'-end of pre-miR-125a, which is associated with several types of cancer, such as gastric and prostate cancer, and glioma. The aim of the present study was to identify the prognostic significance of miR-125a-3p expression levels in patients with NSCLC. The gene expression omnibus database was used to analyze miR-125a-3p expression in NSCLC in silico, and 148 NSCLC samples and 30 adjacent normal lung tissue specimens were analyzed for the expression of miR-125a-3p by qPCR. The results showed that the expression levels of miR-125a-3p in the adjacent normal tissues was higher than the expression level in the NSCLC tissues. There were several clinical parameters demonstrated to be associated with miR-125a-3p expression, such as lymph node metastasis, tumor node metastasis classification of malignant tumor stage and tumor diameter. Furthermore, high expression levels of miR-125a-3p with chemotherapy prolonged the overall survival rate and disease free survival rate compared with untreated patients with low expression of miR-125a-3p. Thus, miR-125a-3p is a significant prognostic biomarker for patients with NSCLC, from which a novel therapeutic strategy to combat NSCLC may be derived.
\end{abstract}

Correspondence to: Professor Da Fu, Department of Nuclear Medicine, Shanghai 10th People's Hospital, Tongji University School of Medicine, 302 Middle Yanchang Road, Zhabei, Shanghai 200072, P.R. China

E-mail: fu800da900@126.com

Dr Chunyan Wu, Department of Pathology, Shanghai Pulmonary Hospital, Tongji University School of Medicine, 507 Zhengming Road, Yangpu, Shanghai 200433, P.R. China

E-mail: chunyan_wu2015@126.com

${ }^{*}$ Contributed equally

Key words: miR-125a-3p, non-small cell lung cancer, chemotherapy, overall survival rate, disease-free survival rate, biomarker, prognosis

\section{Introduction}

Lung cancer is associated with one of the highest mortality rates of all malignant tumors worldwide, resulting in $>1$ million mortalities annually $(1,2)$. Non-small cell lung cancer (NSCLC) is the primary type of lung carcinoma, accounting for $80-85 \%$ of the total number of incidences of lung cancer $(3,4)$. Despite the emergence of novel drugs and therapies, the prognosis of lung cancer remains poor, and the 5-year overall survival (OS) rate is $\sim 11 \%(5,6)$. Therefore, additional analysis of the mechanisms resulting in the occurrence and the development of NSCLC is required to improve the survival rate of lung cancer.

MicroRNAs (miRNAs) are a class of small single stranded non-coding RNAs measuring between 20 and 24 nucleotides (7), which are associated with post-transcriptional gene regulation (8). miRNAs participate in the regulation of cellular proliferation, differentiation and apoptosis by inducing target mRNA degradation or translation inhibition, which affects cancer occurrence and development. Aberrant miRNA expression is associated with the development of different types of cancer. Recent reports (9-11) have suggested that the downregulation of miRNA (miR)-138, miR-218, miR-34c-3p was found in various types of cancer, including NSCLC. Therefore, miRNAs serve an important role in the diagnosis, prediction and treatment of NSCLC and represent novel research targets in the field of molecular biology, which is becoming a prominent discipline within the life sciences.

miR-125a-3p is a member of the miR-125a family, and in the present study has been demonstrated to exert a tumor suppressive effect on several types of tumor, such as glioma (12) and gastric cancer (13). However, there are few studies that demonstrate the link between miR-125a-3p and lung cancer specifically (14). Therefore, the use of miR-125a-3p as a prognostic biomarker for the treatment of NSCLC remains unknown.

The present study explored the differential expression of miR-125a-3p within the gene expression omnibus (GEO) database, and investigated the expression levels of miR-125a-3p in NSCLC tissues using a quantitative polymerase chain reaction (qPCR) assay. Additionally, the present study analyzed the 
association between miR-125a-3p and patient clinicopathological characteristics.

\section{Materials and methods}

Ethical statement. The present study was approved by the Ethics Committee of Shanghai 10th People's Hospital, Tongji University School of Medicine (SHSY-IEC-PAP-15-18; Shanghai, China). All patients provided written informed consent.

Set-up of server for online survival calculation. A total of 4 datasets were used for the miR-125a-3p expression analysis between normal lung and cancer tissues: The expression levels of miR-125a-3p in a number of cells, reported previously, (15) were analyzed; 20 pairs of lung cancer samples and adjacent normal lung samples were compared with GEO datasets, GEO series GSE18692, and 61 lung squamous carcinoma tissues and 10 normal tissues were investigated, GEO series GSE16025. The peripheral miRNA blood profiles from healthy subjects $(n=19)$, lung cancer patients $(n=28)$, and chronic obstructive pulmonary disease (COPD) samples $(n=24)$ had been screened for the complete miRNA repertoire; GEO series GSE24709.

Acquisition of clinical samples. Frozen NSCLC samples $(\mathrm{n}=118)$, and paired carcinoma and adjacent normal lung tissue specimens $(n=30)$ were collected from a total of 148 patients undergoing surgical resection and assessed for the expression level of miR-125a-3p. These tissue samples were collected from Shanghai 10th People's Hospital and Shanghai Pulmonary Hospital, Tongji University School of Medicine (Shanghai, China) between January 2008 and December 2012. The clinical data gathered included the patients' age, gender, smoking history, lymph-node metastasis, tumor differentiation, histological grade, tumor node metastasis (TNM) stage, invasion of lung membrane, vascular invasion, chemotherapy, diameter, OS, disease-free survival (DFS) rate and miR-125a-3p expression status of the patients.

RNA extraction. The RNA, including miRNAs, was extracted from the carcinoma and the adjacent normal lung tissues using TRIzol reagent (Thermo Fisher Scientific, Inc., Waltham, MA, USA) according to protocol of the manufacturer. A Nanodrop 1000 spectrophotometer (Thermo Fisher Scientific Inc., Wilmington, DE, USA) was used to assess the RNA concentration and the purity, following the protocol of the manufacturer. Electrophoresis on 1.5\% denaturing agarose gels was carried out to evaluate the quality of all the RNA specimens.

$R T-q P C R$. For the miR-125a-3p qPCR, complementary DNA was synthesized from $10 \mathrm{ng}$ of the total RNA using the TaqMan Universal PCR kit (cat no. 4304437; Thermo Fisher Scientific, Inc.) and individual miR-125a-3p primers. Specific RT primers and TaqMan probes were used to quantify the expression of hsa-miR-125a-3p (cat no. 4395310). Samples were normalized to RNU6B (cat no. 4373381) as indicated. All reactions, including no-template controls and RT-minus controls, were run in an ABI Prism 7900 HT sequence detection system (Applied Biosystems; Thermo Fisher Scientific,
Inc.). Thermocycling conditions were as follows: Initial denaturation at $94^{\circ} \mathrm{C}$ for $10 \mathrm{~min}$, followed by 35 cycles of $94^{\circ} \mathrm{C}$ for $30 \mathrm{sec}, 60^{\circ} \mathrm{C}$ for $30 \mathrm{sec}$ and $72^{\circ} \mathrm{C}$ for $30 \mathrm{sec}$ with a final extension at $72^{\circ} \mathrm{C}$ for $10 \mathrm{~min}$. Each reaction was independently tested in duplicate a minimum of 3 times. The quantification cycle $(\mathrm{Cq})$ was defined as the fractional cycle number at which the fluorescence passed the fixed threshold. The relative amount of miR-125a-3p to RNU6B was calculated using the equation $2^{-\Delta \Delta \mathrm{Cq}_{\mathrm{q}}}(16)$.

Statistical analysis. All statistical analyses were performed with IBM SPSS 19.0 (IBM Corp., Armonk, NY, USA). The results were presented as the mean \pm standard deviation, using at least 3 independent experiments. A $\chi^{2}$ analysis was carried out to compare the differences between categorical variables and the Wilcoxon signed-rank test was performed to compare of differences between the 2 groups. The Kaplan-Meier method was used to evaluate the univariate probabilities of OS and DFS, and the comparison of the survival curves was assessed using a log rank test. A Cox regression model was used to estimate the univariate and the multivariate hazard ratios. $\mathrm{P}<0.05$ was considered to indicate a statistically significant difference.

\section{Results}

Expression of miR-125a-3p using in silico analysis. Throughcomparing the expression levels of miR-125a-3p indifferent cancer cell lines analyzed in a previous study (15), the present study demonstrated that miR-125a-3p expression levels werelower in the lung cancer A549 cells compared withthe other cancer cell lines, as demonstrated in Fig. 1A. In 20 pairs of lung cancer and adjacent normal lung samples, the expression values in the non-tumoral adjacent $(n=20)$ and in the lung cancer tissues $(n=20)$ were $5.02 \pm 0.49$ and $3.21 \pm 0.58$, respectively compared with the control. This data suggested that the expression of miR-125a wasdownregulated in lung cancer samples $(\mathrm{P}=0.038)$, as illustrated in Fig. 1B. Additionally, the lung squamous carcinoma tissues $(n=61)$ and normal tissues $(n=10)$ were investigated, and these data indicated that miR-125a-3p expression was lower in lung squamous carcinoma tissues, $8.95 \pm 0.16$, in contrast with normal tissues, 10.24 \pm 0.31 , as demonstrated in Fig. $1 \mathrm{C}(\mathrm{P}<0.001)$.

Furthermore, the peripheral miRNA blood profiles from healthy subjects $(n=19)$, patients with lung cancer $(n=28)$, and COPD samples $(n=24)$ werescreened for the complete miRNA repertoire, and their expression values were $61.83 \pm 2.05$, $32.45 \pm 5.75$ and $31.06 \pm 4.53$, respectively. The results revealed that miR-125a-3p was downregulated in lung cancer $(\mathrm{P}=0.027)$, as demonstrated in Fig. 1D.

miRNA-125a-3p expression in NSCLC and normal lung tissue. The levelof miR-125a-3p in 30 paired NSCLC samples were collected and analyzed via RT-qPCR analysis, and it was identified that miR-125a-3p expression levels were lower in lung cancer cells, $2.25 \pm 0.61$, relative to the non-tumor samples, which exhibited levels of $4.01 \pm 0.88(\mathrm{P}=0.048)$ as demonstrated in Fig. 2A. Additionally, the expression levels of miR-125a-3p in the 118 lung cancer samples were examined by RT-qPCR, and are illustrated in Fig. 2B. The expression levels of miR-125a-3p in the adjacent normal tissues, $4.01 \pm 0.88$, 

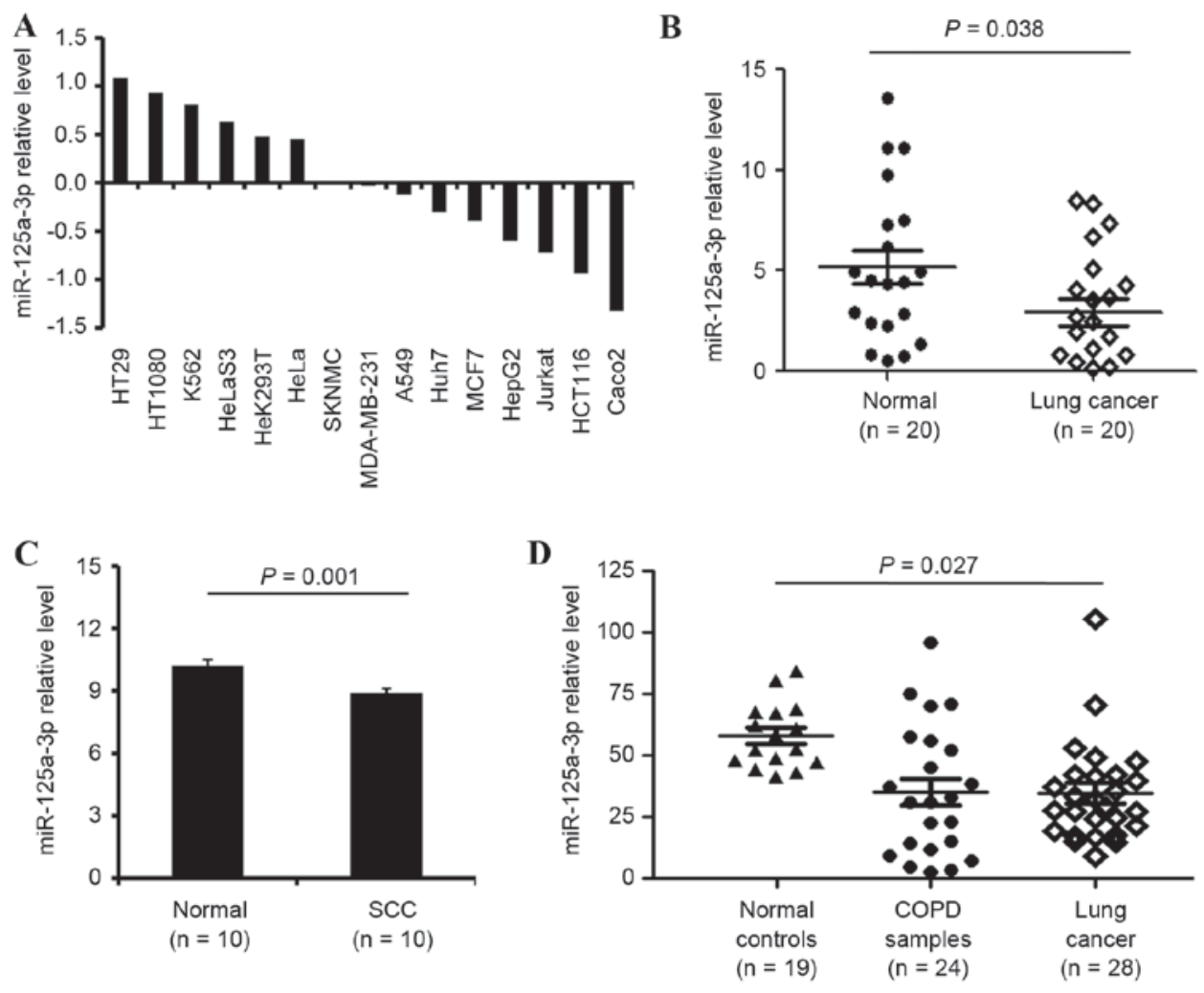

Figure 1. Analysis of miR-125a-3p expression in patients with non-small cell lung cancer with GEO database. (A) Expression levels of miR-125a-3p in 15 cell lines: HT29; HT1080; K562; HeLaS3; HeK293T; HeLa; SKNMC; MDA-MB-231; A549; Huh7; MCF7; HepG2; Jurkat; HCT116; Caco2. (B) Expression levels of miR-125a-3pin normal vs. lung cancer tissues derived from the GEO database, GSE18692. (C) Expression levels of miR-125a-3pin normal vs. lung SCC tissues derived from the GEO database, GSE16025. Levels of miR-125a-3p were summarized and recorded as mean \pm standard deviation. (D) Expression levels of miR-125a-3pin normal vs. COPD vs. lung cancer tissues derived from the GEO database, GSE24709. GEO, gene expression omnibus; SCC, squamous carcinoma cells; COPD, chronic obstructive pulmonary disorder; miR, microRNA.
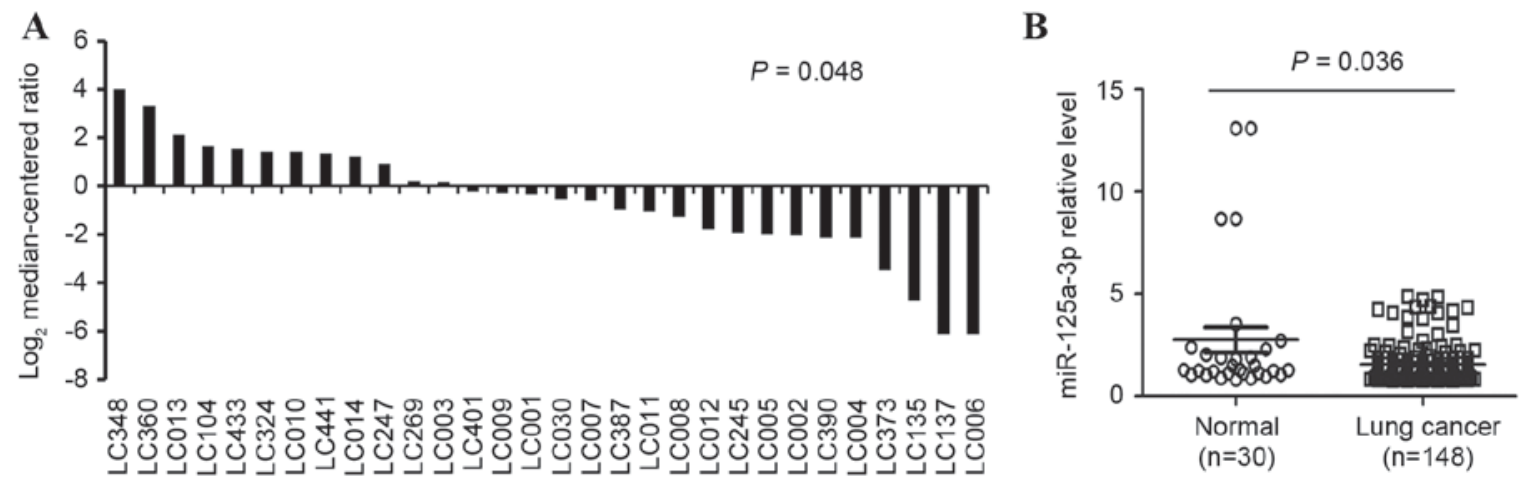

Figure 2. Analysis of miR-125a-3p expression in 30 paired NSCLC tissues by quantitative polymerase chain reaction. (A) Expression levels of miR-125a-3p in 30 paired NSCLC tissues. (B) Expression levels of miR-125a-3p in normal vs. lung cancer tissues. LCXXX, lung cancer sample number XXX; NSCLC, non-small cell lung cancer; miR, microRNA.

weremarkedly higher than the NSCLC tumor biopsies, $1.64 \pm 0.37$. The difference in the expression levels between the normal lung tissue and the NSCLC tumor tissue was statistically significant $(\mathrm{P}=0.036)$.

The association between miR-125a-3p expression and clinical characteristics. Theassociation between miR-125a-3p expression levels and individual clinical characteristics was investigated. A univariate analysis demonstrated that the miR-125a-3p expression level was significantly correlated with lymph-node metastasis $(\mathrm{P}=0.004)$, TNM stage $(\mathrm{P}=0.031)$ and tumor diameter $(\mathrm{P}=0.011)$, as demonstrated in Table $\mathrm{I}$ in all 148 patients with NSCLC. In contrast, no association was identified between age, gender, smoking history, tumor differentiation, histology, invasion of the lung membrane, vascular invasion or chemotherapy $(\mathrm{P}>0.05)$.

Expression of miR-125a-3p was a prognostic marker for the survival of patients with NSCLC. A univariate survival analysis was performed with a Kaplan-Meier test to investigate 
Table I. Univariate analysis of overall survival based on patients stratified by clinical characteristics.

\begin{tabular}{|c|c|c|c|c|}
\hline Factor & Variable & $\mathrm{N}$ & $\begin{array}{c}\text { miR-125a-3p } \\
\text { expression (median) }\end{array}$ & P-value \\
\hline \multirow[t]{2}{*}{ Age (years) } & $\geq 60$ & 87 & 2.78 & \\
\hline & $<60$ & 61 & 3.63 & 0.229 \\
\hline \multirow[t]{2}{*}{ Gender } & Male & 92 & 3.86 & \\
\hline & Female & 56 & 2.99 & 0.192 \\
\hline \multirow[t]{3}{*}{ Smoking history } & Never & 35 & 2.98 & \\
\hline & Ever & 62 & 4.35 & 0.075 \\
\hline & Unknown & 51 & 3.48 & \\
\hline \multirow[t]{3}{*}{ Lymph node metastasis } & Negative & 88 & 6.53 & \\
\hline & Positive & 53 & 4.42 & 0.004 \\
\hline & Unknown & 7 & 5.21 & \\
\hline \multirow[t]{3}{*}{ Tumor differentiation } & Poorly & 52 & 3.69 & \\
\hline & Moderately & 90 & 3.47 & 0.409 \\
\hline & Well & 6 & 5.22 & \\
\hline \multirow[t]{2}{*}{ Histology } & Adenocarcinoma & 50 & 3.85 & \\
\hline & Squamous cell carcinoma & 98 & 5.21 & 0.653 \\
\hline \multirow[t]{2}{*}{ TNM stage } & I-II & 88 & 6.89 & \\
\hline & III-IV & 60 & 4.11 & 0.031 \\
\hline \multirow[t]{3}{*}{ Invasion of lung membrane } & Negative & 31 & 6.15 & \\
\hline & Positive & 109 & 4.23 & 0.063 \\
\hline & Unknown & 8 & 5.21 & \\
\hline \multirow[t]{3}{*}{ Vascular invasion } & Negative & 141 & 3.69 & \\
\hline & Positive & 3 & 2.52 & 0.809 \\
\hline & Unknown & 4 & 3.48 & \\
\hline \multirow[t]{2}{*}{ Diameter $(\mathrm{cm})$} & $\geq 5$ & 36 & 3.22 & \\
\hline & $<5$ & 112 & 6.87 & 0.011 \\
\hline
\end{tabular}

whether several clinical parameters influenced the prognosis of NSCLC. The clinical parameters in the univariate analysis stratification included: Age; gender; smoking history; lymph-node metastasis; tumor differentiation; histology; TNM stage; invasion of the lung membrane; vascular invasion; tumor diameter; and miR-125a-3p expression. The results indicated that tumor size, TNM stage, and lymph-node metastasis were correlated with OS and DFS rates in patients with NSCLC, as illustrated in Fig. 3A and D $(\mathrm{P}<0.05)$. Similarly, miR-125a-3p downregulation was associated with significantly poorer OS rates $(\mathrm{P}=0.012$, and DFS rates $(\mathrm{P}=0.015)$, as demonstrated in Fig. 3E and F, respectively.

A Cox proportional hazards regression model of univariate analysis indicated that the prognosis of thepatients with NSCLC wassignificantly correlated with lymph node metastasis $(\mathrm{P}=0.031)$, TNM stage $(\mathrm{P}=0.035)$ and tumor diameter $(\mathrm{P}=0.005)$ as demonstrated in Table II. However, no correlation was identified between the prognosis and the age, gender, smoking history, tumor differentiation, histology, invasion of lung membrane or vascular invasion values of the patients. Accordingly, the results of the present study demonstrated that the expression levels of miR-125a-3p were associated with the prognosis of patients with NSCLC $(\mathrm{P}=0.012)$, as illustrated in Table II.
To confirm whether the expression levels of miRNA-125a-3p was a prognostic marker in patients with NSCLC, a multivariate analysis with a Cox proportional hazards regression model was performed. Through a series of analyses, the ultimate model of significant predictors of OSrates were defined. These significant predictors included lymph-node metastasis $[\mathrm{P}=0.011 ; \mathrm{HR}=1.989$ (1.346-3.456)], TNM stage $[\mathrm{P}=0.024$; $\mathrm{HR}=1.894$ (1.231-2.265)], tumor diameter $[\mathrm{P}=0.001 ; \mathrm{HR}=3.651$ (2.376-4.335)], as illustrated in Table II. These data identified that high expression levels of miR-125a-3p were a predictor of higher OS rates in patients with NSCLC.

Chemotherapy associated with high expression of miR-125a-3p improves OS andDFS of patients with NSCLC. As demonstrated in Table III, there was a statistically significant association between chemotherapy and $O S$ rates $(27.04 \pm 3.68$ vs. $32.47 \pm 2.98$, $\mathrm{P}=0.008)$ and DFS rates $(26.43 \pm 2.96$ vs. $28.04 \pm 3.36, \mathrm{P}=0.035)$ in patients with NSCLC. Treatment status as a precondition was used to investigate the OS and DFS ratesof patients with NSCLC. According to analysis of the treatment status and expression levels of miR-125a-3p, the present study observed that chemotherapyand high expression levels of miR-125a-3p positively prolongedthe OS, $33.78 \pm 3.94$ vs. $24.32 \pm 2.58$ $(\mathrm{P}=0.001)$, and DFS rates of patients with NSCLC, $30.26 \pm 3.79$ 
Table II. Cox regression model analysis for overall survival based on various clinical characteristics in patients with non-small cell lung cancer.

\begin{tabular}{|c|c|c|c|c|c|c|}
\hline \multirow[b]{2}{*}{ Factor } & \multirow[b]{2}{*}{$\begin{array}{l}\text { Hazard } \\
\text { ratio }\end{array}$} & \multirow[b]{2}{*}{$\begin{array}{c}95 \% \mathrm{CI} \\
\text { (univariate) }\end{array}$} & \multirow[b]{2}{*}{ P-value } & \multicolumn{3}{|c|}{ miR-125a-3p multivariate analysis } \\
\hline & & & & $\begin{array}{c}\text { Hazard } \\
\text { ratio }\end{array}$ & $\begin{array}{c}95 \% \mathrm{CI} \\
\text { (multivariate) }\end{array}$ & P-value \\
\hline Age $(\geq 60$ vs. $<60)$ & 1.274 & $0.698-1.981$ & 0.075 & & & \\
\hline Gender (male vs. female) & 0.811 & $0.533-1.126$ & 0.801 & & & \\
\hline Smoking history (ever vs. never) & 0.991 & $0.423-1.887$ & 0.186 & & & \\
\hline Lymph-node metastasis (positive vs. negative) & 1.737 & $1.186-2.951$ & 0.031 & 1.989 & $1.346-3.456$ & 0.011 \\
\hline Tumor differentiation (poorly vs. well) & 0.754 & $0.461-1.234$ & 0.261 & & & \\
\hline Histology (adenoma. vs. squamous) & 0.775 & $0.482-1.247$ & 0.294 & & & \\
\hline Tumor node metastasis stage (III-IV vs. I-II) & 1.683 & $1.116-2.095$ & 0.035 & 1.894 & $1.231-2.265$ & 0.024 \\
\hline Invasion of lung membrane (positive vs. negative) & 1.221 & $1.026-1.434$ & 0.525 & & & \\
\hline Vascular invasion (positive vs. negative) & 0.989 & $0.683-1.265$ & 0.317 & & & \\
\hline Diameter ( $\geq 5$ vs. $<5 \mathrm{~cm})$ & 2.382 & $1.305-4.346$ & 0.005 & 3.651 & $2.376-4.335$ & 0.001 \\
\hline Chemotherapy (positive vs. negative) & 0.775 & $0.428-1.122$ & 0.029 & 0.536 & $0.359-0.721$ & 0.004 \\
\hline miR-125a-3p expression (high vs. low) & 0.671 & 0.423-0.989 & 0.012 & & & \\
\hline
\end{tabular}

CI, confidence interval; miR; microRNA.
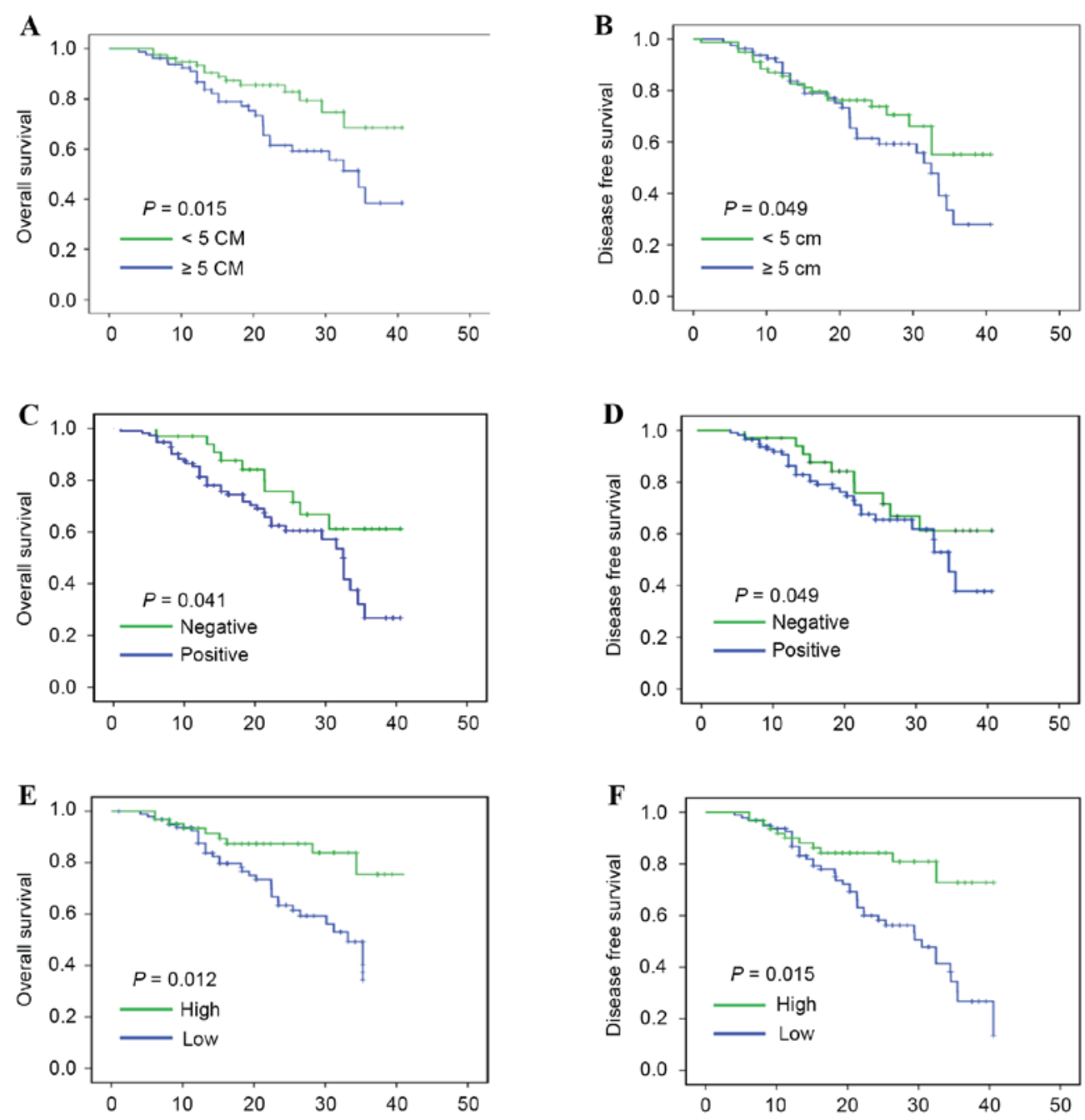

Figure 3. Univariate survival analysis of different clinical parameters in NSCLC. Univariate survival analysis of overall survival and disease-free survival rates in lung carcinoma as determined by Kaplan-Meier plot estimates based on diameter in (A and B), lymph node metastasis in (C and D) and microRNA-125a-3p expression in $(\mathrm{E}$ and $\mathrm{F})$. 
Table III. OS and DFS of patients with non-small cell lung cancer based on chemotherapy alone vs. chemotherapy and miR-125a-3p expression.

\begin{tabular}{|c|c|c|c|c|c|c|}
\hline \multirow[b]{2}{*}{ Factor } & \multirow[b]{2}{*}{ Median } & \multicolumn{2}{|l|}{ OS } & \multicolumn{2}{|r|}{ DFS } & \multirow[b]{2}{*}{ P-value } \\
\hline & & $95 \%$ CI & P-value & Median & $95 \% \mathrm{CI}$ & \\
\hline \multicolumn{7}{|c|}{ Chemotherapy } \\
\hline Positive & 32.47 & 26.78-34.69 & 0.008 & 28.04 & $26.54-30.18$ & 0.035 \\
\hline Negative & 27.04 & $23.43-29.06$ & & 26.43 & 24.31-28.69 & \\
\hline \multicolumn{7}{|c|}{ Chemotherapy and expression } \\
\hline $\mathrm{P} \& \mathrm{H}$ & 33.78 & $31.49-35.74$ & 0.001 & 30.26 & $26.18-32.54$ & $<0.001$ \\
\hline$N \& L$ & 24.32 & $18.94-26.03$ & & 22.04 & $19.43-24.65$ & \\
\hline
\end{tabular}

P \& H, chemotherapy and miR-125a-3p high expression; N \& L, no chemotherapy and miR-125a-3p low expression; overall survival rate; DFS, disease-free survival rate.
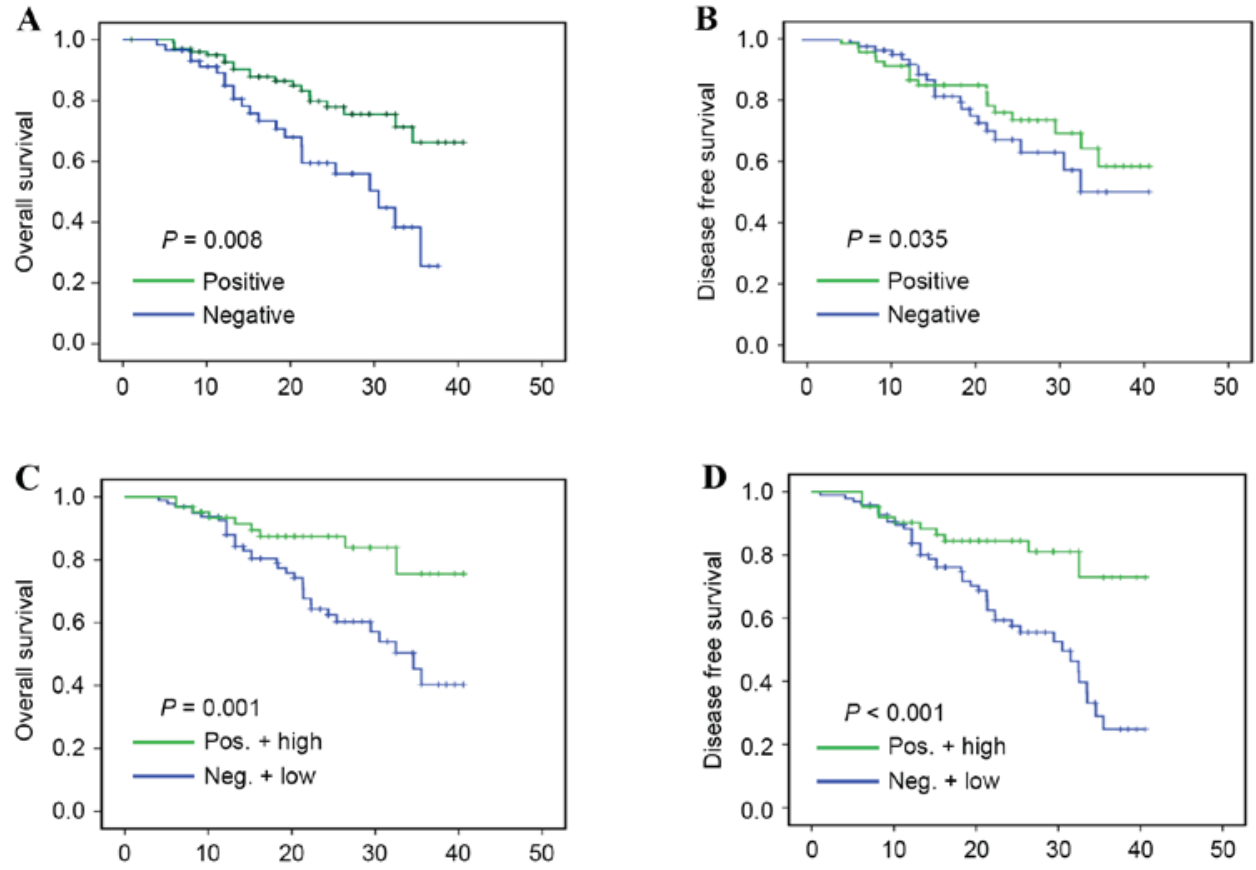

Figure 4. Survival rate analysis of chemotherapy and miR-125a expression levels in Non-small cell lung cancer. OS (A) and DFS (B) rates in lung carcinoma determined by Kaplan-Meier plot estimates based on chemotherapy. (C) OS and (D) DFS rates in lung carcinoma as determined by Kaplan-Meier plots estimates based on chemotherapy and miR-125a-3p expression. OS, overall survival; DFS, disease-free survival; miR, microRNA.

vs. 22.04 $\pm 2.86(\mathrm{P}<0.001)$, comparingpatients with NSCLC administered non-chemotherapy treatments and low expression levels of miR-125a-3p, as outlined in Table III.

To confirm the association between miR-125a-3p expression levels and chemotherapy with OS and DFS rates, univariate and multivariate survival analyses conducted with Kaplan-Meier estimates were carried out. The univariate analysis demonstrated that chemotherapy was associated with prognosis $(\mathrm{P}=0.029$; $\mathrm{HR}=0.775$ [0.428-1.122]), as illustrated in Table II, and the multivariate survival analysis indicated that a high expression level of miR-125a-3p with chemotherapy was associated with prognosis $[\mathrm{P}=0.004$; $\mathrm{HR}=0.536$ (0.359-0.721), also illustrated in Table II]. In addition, the results showed that chemotherapy was positively correlated with OS $(\mathrm{P}=0.008)$, and DFS rates $(\mathrm{P}=0.035)$, as demonstrated in Fig. 4A and B, respectively. Furthermore, a high expression level of miR-125a-3p in patients with NSCLC who had undergone chemotherapy was also positively correlated with OS $(\mathrm{P}=0.001)$, and DFS rates $(\mathrm{P}<0.001)$, as illustrated in Fig. 4C and D, respectively, which revealed that patients with NSCLC with high expression levels of miR-125a-3p may be more likely to benefit from chemotherapy.

\section{Discussion}

The present study revealed that miR-125a-3p expression levels were lower in the lung cancer A549 cells compared with the other cancer cell lines, and it is also downregulated in NSCLC tissues when compared with normal lung tissues. The OS 
and DFS rates of patients with low miR-125a-3p expression levels were higher compared with patients exhibiting high miR-125a-3p expression levels. The analysis of several clinical factors suggested that lymph node metastasis and tumor diameter were associated with poor prognoses in NSCLC. Notably, miR-125a-3p expression levels as an independent parameter also affected prognosis and survival. In addition, high expression levels of miR-125a-3p combined with chemotherapy resulted in high OS rates compared with the low expression levels of miR-125a-3p with no chemotherapy. Therefore, miR-125a-3p may act as a biomarker for chemotherapy in NSCLC.

miR-125a is a type of miRNA that has been demonstrated to be associated with numerous types of cancer. According to the $5^{\prime}$ and $3^{\prime}$ ends of pre-miR-125a, the miR-125a family may be divided into different members, including miR-125a-5p and $\mathrm{miR}-125 \mathrm{a}-3 \mathrm{p}$ (17). There are several reports suggesting that miR-125a-5p is specifically downregulated in breast and liver cancer, melanoma and bladder and colon cancer (18-21). In addition, previous studies have revealed the dysregulated expression of miR-125a-3p in several types of cancer, for example, miR-125a-3p was downregulated in malignant glioma (12) and prostate (22), gastric (13) and pancreatic (23) cancer. Inversely, miR-125a-3p was significantly upregulated in synovial (24) and rectal carcinoma (25) and multiple myeloma (26). Furthermore, a previous study revealed that miR-125a-3p is over-expressed in retinoblastoma under hypoxic conditions (27). miR-125a-3p has been demonstrated to exhibit a strong association with a regulatory pathway of lung cancer: Jiang et al (28) demonstrated that miR-125a-3p functioned as a lung carcinoma suppressor gene that inhibited A549 cell proliferation and apoptosis, and indicated that the p53 pathway is not the only pathway involved in inducing lung cancer cell apoptosis. Previous studies have also suggested miR-125a-3p participates in the development of lung cancer via the classical mitogen-activated protein kinase signaling pathway (29) and the Ras homolog gene family, member A-actomyosin pathway (30). A previous study indicated that miR-125a-3p suppresses NSCLC cell proliferation, migration and invasion through the binding of miR-125a-3p to the 3'-UTR of metastasis-associated gene 1 (MTA1) (31). Through the aforementioned analysis, the present study identified that miR-125a-3p suppresses proliferation, metastasis and apoptosis in lung cancer via numerous pathways, and participates in the regulation of carcinoma development. Therefore, the development of novel targets of the miR-125a-3p gene that improve the treatment of NSCLC requires attention. miR-125a-3p exhibits a strong association with NSCLC, and it serves an important role in the molecular therapy of NSCLC.

The present study revealed a series of molecules that are closely associated with carcinoma development. These characteristic molecules are involved in the treatment of cancer; as such, the role of biomarkers in the clinical diagnosis and prognosis of patients with cancer is critical. Previous reports have demonstrated that miRNAs are underlying diagnostic biomarkers and prognostic factors for lung carcinoma $(32,33)$. Several miRNAs such as miR-25 (34), miR-9 (35), miR-498 (36) have been revealed to exhibit clinical value in the diagnosis of NSCLC. In addition, these novel biomarkers may possibly promote innovative therapeutic methods using novel drug compounds.

miRNAs are useful prognostic predictors and therapeutic targets within clinical chemotherapy. The analysis of the signal pathways associated with cancer and the predication of prognosis subsequent to chemotherapy depends on the emergence of individual biomarkers in patients with NSCLC. Thus, the identification of unique molecular biomarkers is required to increase the level of early diagnosis and improve the prognosis of chemotherapy. At present, efforts are being made to determine biomarkers with diagnostic and prognostic significance for NSCLC.

In summary, the results of the present study revealed that patients who received chemotherapy exhibited higher OS and DFS rates, compared with untreated ones. additionally, patients with NSCLC with high expression levels of miR-125a-3p who were administered chemotherapy demonstrated longer OS and DFS rates, relative to the untreated patients with low expression levels of miR-125a-3p. Therefore, miR-125a-3p appears to be downregulated in NSCLC, which increased OS and DFS rates of patients receiving chemotherapy. The data of the present study indicates that miR-125a-3p serves as an independent prognostic biomarker for chemotherapy within clinical practice. However, additional studies are required in this field.

The results from the present study also indicated that the expression levels of miR-125a-3p in normal tissues were higher than in NSCLC tissues. In addition, analysis suggested that patients with NSCLC with high expression levels of miR-125a-3p have an improved prognosis, and may be more likely to respond well to chemotherapy. In conclusion, the present study demonstrated that miR-125a-3p is a significant prognostic biomarker for NSCLC, from which novel therapeutic strategies to combat NSCLC may be derived.

\section{Acknowledgements}

The present study was supported by grants from the National Natural Science Foundation of China (grant nos. 81372175, 81472501, 81472202 and 81302065), Shanghai Municipal Commission of Health and Family Planning (grant no. 201440398) and Shanghai Natural Science Foundation (grant no. 16ZR1428900).

\section{References}

1. Han Y, Jia C, Cong X, Yu F, Cai H, Fang S, Cai L, Yang H, Sun Y, Li D, et al: Increased expression of TGF $\beta$ R2 is associated with the clinical outcome of non-small cell lung cancer patients treated with chemotherapy. PLoS One 10: e0134682, 2015.

2. Meng W, Ye Z, Cui R, Perry J, Dedousi-Huebner V, Huebner A, Wang Y, Li B, Volinia S, Nakanishi H, et al: MicroRNA-31 predicts the presence of lymph node metastases and survival in patients with lung adenocarcinoma. Clin Cancer Res 19: 5423-5433, 2013.

3. Mitra R, Edmonds MD, Sun J, Zhao M, Yu H, Eischen CM and Zhao Z: Reproducible combinatorial regulatory networks elucidate novel oncogenic microRNAs in non-small cell lung cancer. RNA 20: 1356-1368, 2014.

4. Zhang L, Wang XF, Ma YS, Xia Q, Zhang F, Fu D and Wang YC: Quantitative assessment of the influence of TP63 gene polymorphisms and lung cancer risk: Evidence based on 93,751 subjects. PLoS One 9: e87004, 2014.

5. Shen S, Yue H, Li Y, Qin J, Li K, Liu Y and Wang J: Upregulation of miR-136 in human non-small cell lung cancer cells promotes Erk1/2 activation by targeting PPP2R2A. Tumor Biol 35: 631-640, 2014. 
6. Fang Y, Fu D and Shen XZ: The potential role of ubiquitin c-terminal hydrolases in oncogenesis. Biochim Biophys Acta 1806: 1-6, 2010

7. Agirre X, Vilas-Zornoza A,Jiménez-Velasco A, Martin-Subero JI, Cordeu L, Gárate L, San José-Eneriz E, Abizanda G, Rodríguez-Otero P, Fortes P, et al: Epigenetic silencing of the tumor suppressor microRNA hsa-miR-124a regulates CDK6 expression and confers a poor prognosis in acute lymphoblastic leukemia. Cancer Res 69: 4443-4453, 2009.

8. Pierson J, Hostager B, Fan R and Vibhakar R: Regulation of cyclin dependent kinase 6 by microRNA 124 in medulloblastoma. J Neurooncol 90: 1-7, 2008.

9. Li JQ, Hu SY, Wang ZY, Lin J, Jian S, Dong YC, Wu XF, Lan D and Cao LJ: MicroRNA-125-5p targeted CXCL13: A potentia biomarker associated with immune thrombocytopenia. Am J Transl Res 15: 772-780, 2015.

10. Xie J, Yu F, Li D, Zhu X, Zhang X and Lv Z: MicroRNA-218 regulates cisplatin (DPP) chemosensitivity in non-small cell lung cancer by targeting RUNX2. Tumor Biol 37: 1197-1204, 2016.

11. Zhou YL, Xu YJ and Qiao CW: miR-34c-3p suppresses the proliferation and invasion of non-small cell lung cancer (NSCLC) by inhibiting PAC1/MAPK pathway. Int J Clin Exp Pathol 8: 6312-6322, 2015

12. Wang H, Xu T, Jiang Y, Xu H, Yan Y, Fu D and Chen J: The challenges and the promise of molecular targeted therapy in malignant gliomas. Neoplasia 17: 239-255, 2015.

13. Hashiguchi Y, Nishida N, Mimori K, Sudo T, Tanaka F, Shibata K, Ishii H, Mochizuki H, Hase K, Doki Y and Mori M: Down-regulation of miR-125a-3p in human gastric cancer and its clinicopathological significance. Int J Oncol 40: 1477-1482, 2012.

14. Ruike Y, Ichimura A, Tsuchiya S, Shimizu K, Kunimoto R, Okuno Y and Tsujimoto G: Global correlation analysis for micro-RNA and mRNA expression profiles in human cell lines. J Hum Genet 53: 515-523, 2008

15. Zhang H, Zhu X, Li N, Li D, Sha Z, Zheng $X$ and Wang $H$ : miR-125a-3p targets MTA1 to suppress NSCLC cell proliferation, migration, and invasion. Acta Biochim Biophys Sin (Shanghai) 47: 496-503, 2015.

16. Raponi M, Dossey L, Jatkoe T, Wu X, Chen G, Fan H and Beer DG: MicroRNA classifiers for predicting prognosis of squamous cell lung cancer. Cancer Res 69: 5776-5783, 2009.

16. Livak KJ and Schmittgen TD: Analysis of relative gene expression data using real-time quantitative PCR and the 2(-Delta Delta C(T)) method. Methods 25: 402-408, 2001

17. Jiang L, Huang Q, Zhang S, Zhang Q, Chang J, Qiu X and Wang E: hsa-miR-125a-3p and hsa-miR-125a-5p are downregulated in non-small cell lung cancer and have inverse effects on invasion and migration of lung cancer cells. BMC Cancer 10 $318,2010$.

18. Li W, Duan R, Kooy F, Sherman SL, Zhou W and Jin P: Germline mutation of microRNA-125a is associated with breast cancer. J Med Genet 46: 358-360, 2008.

19. Bi Q, Tang S, Xia L, Du R, Fan R, Gao L, Jin J, Liang S, Chen Z, $\mathrm{Xu}$ G, et al: Ectopic expression of miR-125a inhibits the proliferation and metastasis of hepatocellular carcinoma by targeting MMP11 and VEGF. PLoS One 7: e40169, 2012.

20. Huang L, Luo J, Cai Q, Pan Q, Zeng H, Guo Z, Dong W, Huang J and Lin T: MicroRNA-125b suppresses the development of bladder cancer by targeting E2F3. Int J Cancer 128: 1758-1769, 2011.

21. Tong Z, Liu N, Lin L, Guo X, Yang D and Zhang Q: miR-125a-5p inhibits cell proliferation and induces apoptosis in colon cancer via targeting BCL2, BCL2L12 and MCL1. Biomed Pharmacother 75: 129-136, 2015.
22. Ninio-Many L, Grossman H, Levi M, Zilber S, Tsarfaty I, Shomron N, Tuvar A, Chuderland D, Stemmer SM, Ben-Aharon I and Shalgi R: MicroRNA miR-125a-3p modulates molecular pathway of motility and migration in prostate cancer cells Oncoscience 1: 250-261, 2014.

23. Kojima M, Sudo H, Kawauchi J, Takizawa S, Kondou S, Nobumasa $\mathrm{H}$ and Ochiai A: MicroRNA markers for the diagnosis of pancreatic and biliary-tract cancers. PLoS One 10: e0118220, 2015.

24. Hisaoka M, Matsuyama A, Nagao Y, Luan L, Kuroda T, Akiyama $\mathrm{H}$, Kondo $\mathrm{S}$ and Hashimoto $\mathrm{H}$ : Identification of altered microRNA expression patterns in synovial sarcoma. Genes Chromosomes Cancer 50: 137-145, 2011.

25. Della VittoriaScarpati G, Falcetta F, Carlomagno C, Ubezio P, Marchini S, De Stefano A, Singh VK, D'Incalci M, De Placido S and Pepe S: A specific miRNA signature correlates with complete pathological response to neoadjuvant chemoradiotherapy in locally advanced rectal cancer. Int J Radiat Oncol Biol Phys 83: 1113-1119, 2012.

26. Chonglei Bi, Chung TH, Huang G, Zhou J, Yan J, Gregory JA, Fonseca $\mathrm{R}$ and Chng WJ: Genome-wide pharmacologic unmasking identifies tumor suppressive microRNAs in multiple myeloma. Oncotarget 6: 26508-26518, 2015.

27. Xu X, Jia R, Zhou Y, Song X, Wang J, Qian G, Ge S and Fan X: Microarray-based analysis: Identification of hypoxia-regulated microRNAs in retinoblastoma cells. Int J Oncol 38: 1385-1393, 2011.

28. Jiang L, Chang J, Zhang Q, Sun L and Qiu X: MicroRNA hsa-miR-125a-3p activates p53 and induces apoptosis in lung cancer cells. Cancer Invest 31: 538-544, 2013.

29. Zhou DH, Wang $X$ and Feng Q: EGCG enhances the efficacy of cisplatin by downregulating hsa-miR-98-5p in NSCLC A549 cells. Nutr Cancer 66: 636-644, 2014.

30. Huang B, Luo W, Sun L, Zhang Q, Jiang L, Chang J, Qiu X and Wang E: miRNA-125a-3p is a negative regulator of the RhoA-actomyosin pathway in A549 cells. Int J Oncol 42: 1734-1742, 2013.

31. Zhang H, Zhu X, Li N, Li D, Sha Z, Zheng X and Wang H: miR-125a-3p targets MTA1 to suppress NSCLC cell proliferation, migration and invasion. Acta Biochim Biophys Sin (Shanghai) 47: 496-503, 2015.

32. Li M, Zhang Q, Wu L, Jia C, Shi F, Li S, Peng A, Zhang G, Song X and Wang C: Serum miR-499 as a novel diagnostic and prognostic biomarker in non-small cell lung cancer. Oncol Rep 31: 1961-1967, 2014

33. Zhou DH, Wang X and Feng Q: EGCG enhances the efficacy of cisplatin by downregulating hsa-miR-98-5p in NSCLC A549 cells. Nutr Cancer 66: 636-644, 2014.

34. Xu FX, Su YL, Zhang H, Kong JY, Yu H and Qian BY: Prognostic implications for high expression of miR-25 in lung adenocarcinomas of female non-smokers. Asian Pac J Cancer Prev 15: 1197-1203, 2014.

35. Xu T, Liu X, Han L, Shen H, Liu L and Shu Y: Up-regulation of miR-9 expression as a poor prognostic biomarker in patients with non-small cell lung cancer. Clin Transl Oncol 16: 469-475, 2013

36. Wang M, Zhang Q, Wang J and Zhai Y: MicroRNA-498 is downregulated in non-small cell lung cancer and correlates with tumor progression. J Cancer Res Ther 11 (Suppl 1): S107-S111, 2015. 